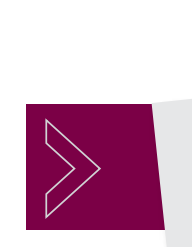

- Syncopal episode

- Chest pain

- Mild lightheadedness

\section{Michael A. Chen, MD, PhD \\ Harborview Medical Center, University of Washington School of Medicine, Seattle \\ vmichen@u. washington.edu}

The author reported no potential conflict of interest relevant to this article.

doi: $10.12788 / \mathrm{jfp} .0331$

A 52-year-old man with a history of hypertension and gastroesophageal reflux disease (GERD) presented to the emergency department (ED) after an episode of syncope. He reported that the syncope occurred soon after he stood up to go to the kitchen to make dinner but was without prodrome or associated symptoms. He recalled little of the event, and the episode was unwitnessed. He had a few bruises on his arms but no significant injuries.

On questioning, he reported occasional palpitations but no changes in his normal exercise tolerance. His only medication was lisinopril $10 \mathrm{mg} / \mathrm{d}$.

In the ED, his vital signs, physical exam (including orthostatic vital signs), basic labs (including troponin I), and a 12-lead EKG were normal. After a cardiology consultation, he was discharged home with a 30-day ambulatory rhythm monitor.

A few days later, while walking up and down some hills, he experienced about 15 seconds of chest pain accompanied by mild lightheadedness. Thinking it might be related to his GERD, he took some over-the-counter antacids when he returned home, since these had been effective for him in the past.

However, the rhythm monitoring company contacted the EKG lab to transmit a concerning strip (FIGURE). They also reported that the patient had been contacted and reported no further symptoms.

\title{
THE DIAGNOSIS
}

Most notable on the patient's rhythm strip was a continuously varying QRS complex, which was indicative of polymorphic ventricular tachycardia and consistent with the patient's syncope and other symptoms. Less obvious at first glance was an STsegment elevation in the preceding beats. Comparison to a post-episode tracing (FIGURE) highlights the abnormality. Polymorphic ventricular tachycardia resolves in 1 of 2 ways: It will either stop on its own (causing syncope if it lasts more than a few seconds) or it will devolve into ventricular fibrillation, causing cardiac arrest. ${ }^{1}$

The combination of these findings and the clinical scenario prompted a recommendation that the patient report to

\section{FIGURE}

A "concerning" rhythm strip prompted action

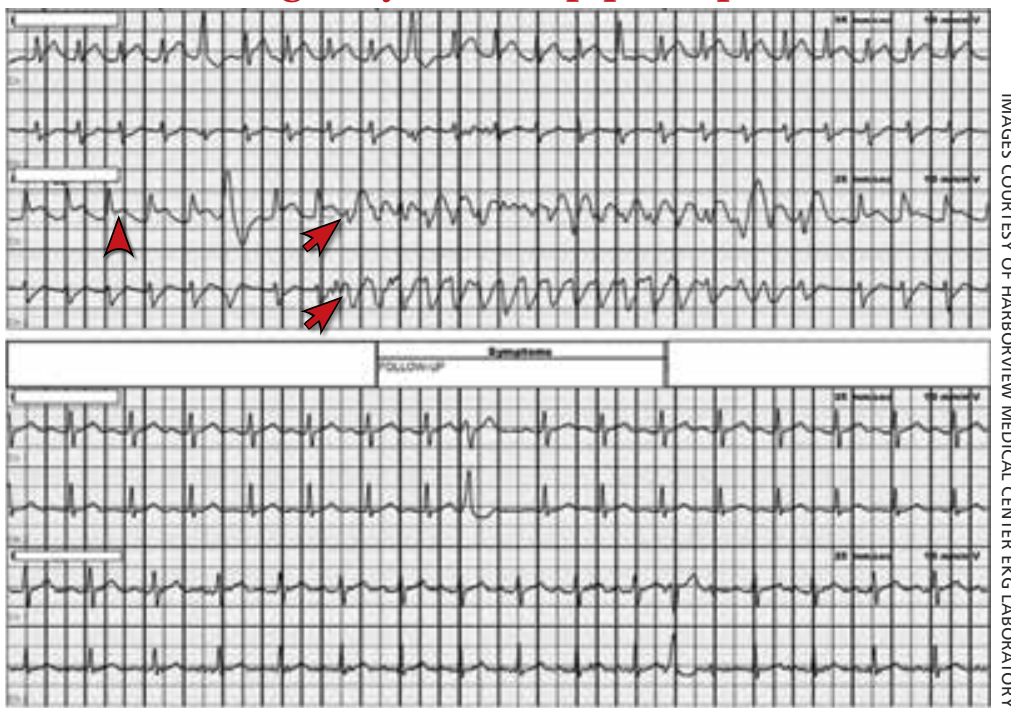

Cardiac monitoring during the patient's episode of chest pain (upper panel) captured a sequence of polymorphic ventricular tachycardia (arrows) and an ST-segment elevation (arrowhead). After the episode, the rhythm strip demonstrated a resolution of the STsegment elevation (lower panel). 


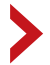

Our patient experienced syncope upon standing, which suggested a noncardiac cause. However, his history of palpitations increased our suspicion for a cardiac cause. the ED for admission (his wife drove him). $\mathrm{He}$ was admitted to the intensive care unit (ICU) for continuous telemetry monitoring, and a cardiac catheterization was ordered. The procedure revealed a $99 \%$ thrombotic mid-right coronary artery lesion, for which aspiration thrombectomy and uncomplicated stenting were performed.

\section{DISCUSSION}

Guidelines from the American College of Cardiology/American Heart Association/ Heart Rhythm Society recommend a detailed history and physical exam, as well as an EKG, for the initial evaluation of syncope. ${ }^{2}$ If this does not point to a diagnosis (and depending on the presentation and other factors), an ambulatory rhythm monitor can be considered. Other possible testing modalities include stress testing, resting transthoracic echocardiography, electrophysiologic testing, and cardiac magnetic resonance imaging or computed tomography.

IIs the cause cardiac? The guidelines suggest that a cardiac cause of syncope is more likely if several of the following factors are present: age $>60$ years; male sex; presence of known heart disease (acquired or congenital); brief prodrome (eg, palpitations) or no prodrome; exertional or supine syncope; 1 to 2 episodes; an abnormal cardiac exam; and a family history of premature sudden death. ${ }^{2}$ A noncardiac cause is suggested by other factors: younger age; no known cardiac disease; standing or a position change from supine to sitting/standing; prodrome; specific triggers (eg, dehydration, pain); and frequent and prolonged stereotypic episodes. $^{2}$

While the guidelines do not specify the number of factors or endorse a specific scoring system, such tools have been developed. For example, the EGSYS (Evaluation of Guidelines in Syncope Study) Score assigns 1 point for each of 6 factors: palpitations; heart disease and/or abnormal EKG; effort syncope; supine syncope; precipitating or predisposing factors; and autonomic prodromes. A score $\geq 3$ identified cardiac syncope with a sensitivity of $95 \%$, but with a specificity of only $61 \%$. In the derivation study, patients with a score $\geq 3$ had higher mortality than those with a lower score ( 17 vs $3 \% ; P<.001){ }^{3}$

I Myocardial ischemia can trigger ventricular arrhythmias. In the GUSTO-1 trial of fibrinolytic therapy in patients with acute ST-segment elevation myocardial infarction $(n=40,895)$, the incidence of ventricular tachycardia or ventricular fibrillation was $10.2 \%{ }^{4}$ In a pooled analysis (4 trials; $\mathrm{n}=26,416)$ of patients who were treated for non-ST-segment elevation or unstable angina-type acute coronary syndromes, the rate of these arrhythmias was markedly lower (2.1\%). ${ }^{5}$ The risk of ventricular arrhythmia is one reason close monitoring (eg, continuous telemetry, ICU admission) is the standard of care for patients with acute coronary syndromes.

I Our patient experienced syncope upon standing, which suggested a noncardiac cause (usually orthostatic hypotension). However, the history of palpitations increased the suspicion for a cardiac cause, and thus the rhythm monitor was ordered.

\section{THE TAKEAWAY}

This case was unusual in that ambulatory monitoring captured electrocardiographic evidence of myocardial ischemia leading directly to a ventricular arrhythmia. In the evaluation of syncope, a detailed history, physical exam, and a baseline 12-lead EKG can sometimes give clues to an arrhythmic cause of syncope (eg, Brugada syndrome, prior infarct pattern, prolonged QTc, bradycardia, heart block, arrhythmogenic right ventricular cardiomyopathy)-but prolonged rhythm monitoring is sometimes needed to identify a cause.

JFP

\section{CORRESPONDENCE}

Michael A. Chen, MD, PhD, Harborview Medical Center, University of Washington School of Medicine, 325 9th Avenue, Box 359748 (Cardiology), Seattle, WA 98104; michen@u.washington.edu

\footnotetext{
References

1. Viskin S, Chorin E, Viskin D, et al. Polymorphic ventricular tachycardia: terminology, mechanism, diagnosis, and emergency therapy. Circulation. 2021;144:823-839. doi: 10.1161/ CIRCULATIONAHA.121.055783

2. Shen W-K, Sheldon RS, Benditt DG, et al. 2017 ACC/AHA/HRS CONTINUED ON PAGE 38
} 
CONTINUED FROM PAGE 36

guideline for the evaluation and management of patients with syncope: executive summary: a report of the American College of Cardiology/American Heart Association Task Force on Cliniof Cardiology/American Heart Association Task Force on Clini-
cal Practice Guidelines and the Heart Rhythm Society. J Am Coll Cardiol. 2017;70:620-663. doi: 10.1016/j.jacc.2017.03.002

3. Del Rosso A, Ungar A, Maggi R, et al. Clinical predictors of cardiac syncope at initial evaluation in patients referred urgently to a general hospital: the EGSYS score. Heart. 2008;94:1528-1529. doi: $10.1136 /$ hrt.2008.143123
4. Newby KH, Thompson T, Stebbins A, et al. Sustained ventricular arrhythmias in patients receiving thrombolytic therapy: incidence and outcomes. The GUSTO Investigators. Circulation. 1998;98:2567-2573. doi: 10.1161/01.cir.98.23.2567

5. Al-Khatib SM, Granger CB, Huang Y, et al. Sustained ventricular arrhythmias among patients with acute coronary syndromes with no ST-segment elevation: incidence, predictors, and outcomes. Circulation. 2002;106:309-12. doi: 10.1161/ 01.cir.0000022692.49934.e3 